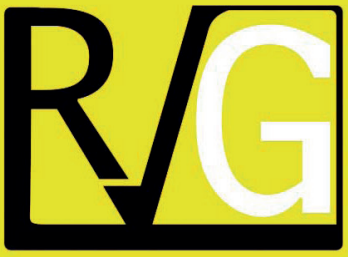

Julio - Septiembre, 2021

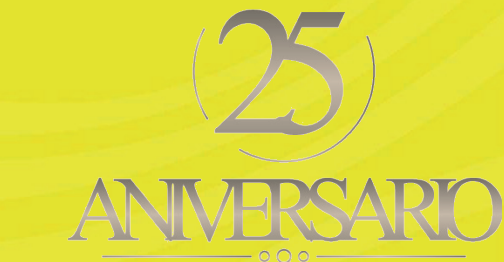

ANMERSARO
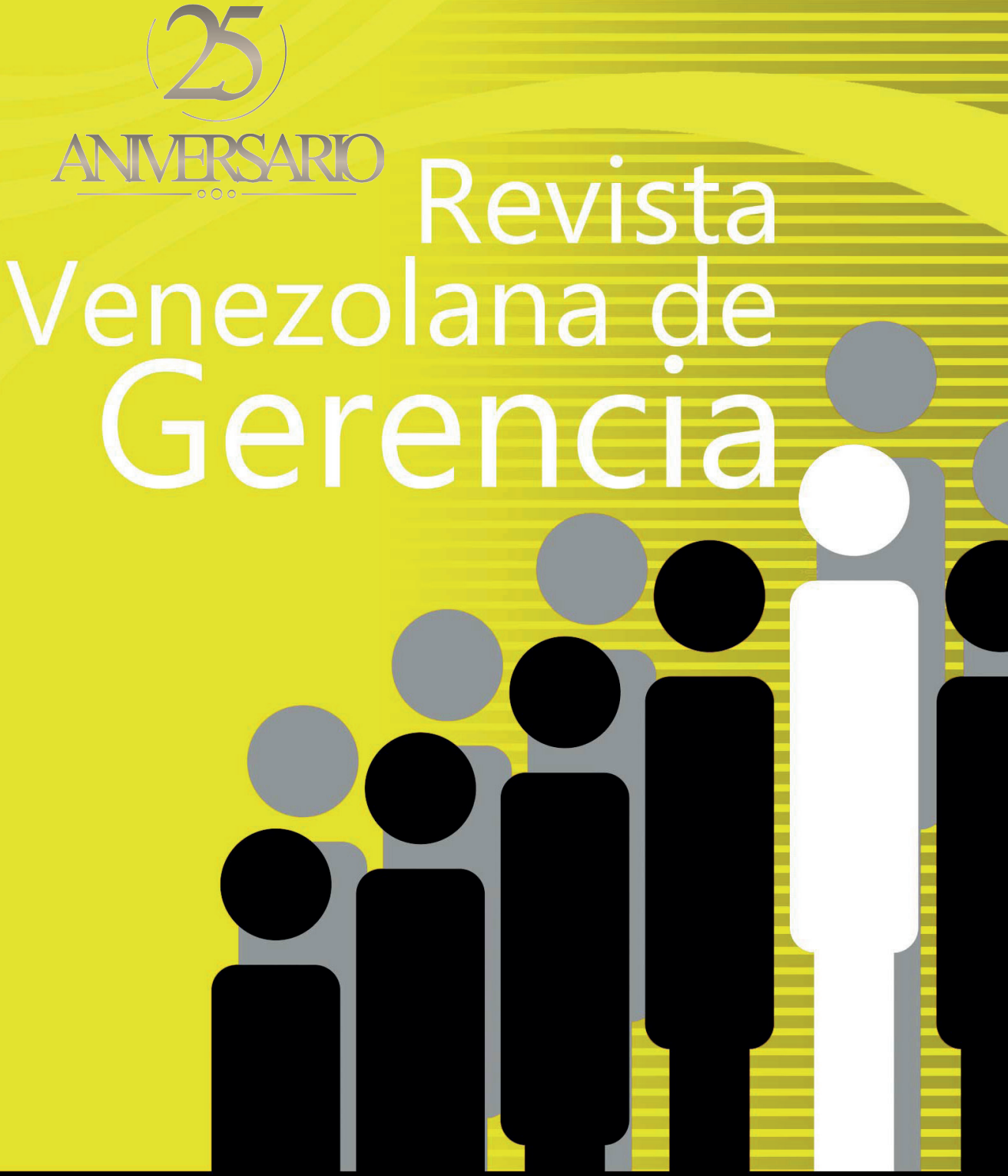

UNIVERSIDAD DEL ZULIA (LUZ)

Facultad de Ciencias Económicas y Sociales Centro de Estudios de la Empresa

ISSN 1315-99

Esta obra está bajo una licencia de Creative Comm Reconocimiento-NoComercial-Compartirlgual 3.0 Unpo http://creativecommons.org/licenses/by-nc-sa/3.0/deed.es 
COMO CITAR: Cueva Estrada, J. M., Sumba Nacipucha, N. A., y Delgado Figueroa, S. P. (2021). Marketing social y su incidencia en el comportamiento del consumidor. Revista Venezolana de Gerencia (RVG), 26(95), 852$867 . \quad$ https://doi.org/10.52080/

\author{
Universidad del Zulia (LUZ) \\ Revista Venezolana de Gerencia (RVG) \\ Año 26 No. 95 Julio-Septiembre 2021, 852-867 \\ ISSN 1315-9984 / e-ISSN 2477-9423
}

\title{
Marketing social y su incidencia en el comportamiento del consumidor
}

\author{
Cueva Estrada, Jorge Manuel* \\ Sumba Nacipucha, Nicolás Armando** \\ Delgado Figueroa, Stella Paola***
}

\section{Resumen}

El presente artículo busca determinar los efectos del marketing social en el comportamiento de compra del consumidor. Para ello, se empleó el cuestionario como instrumento de recolección de datos, esta herramienta se sometió a un test de fiabilidad obteniendo un alfa de Cronbach de 0.815 , la cual fue aplicada a 432 ecuatorianos. A continuación, se desarrolló un estudio correlacional entre las variables, el resultado de la investigación demuestra la existencia de correlación de intensidad baja (rho de Spearman $=.322$ ), resultado que corrobora que el marketing social no tiene como fin último motivar o incidir positivamente en la decisión de compra del consumidor, sobre productos que comercializa la empresa, no obstante, si favorece la imagen corporativa.

Palabras clave: Comportamiento de compra; consumidor; imagen corporativa; marketing social.

* Máster en Administración de Empresas. Profesor-Investigador de la carrera administración de empresas de la Universidad Politécnica Salesiana del Ecuador sede Guayaquil. Editor de Revista RETOS y miembro de Grupo de Innovación Educativa Mejorando las Prácticas Áulicas (GIE-MPA), Universidad Politécnica Salesiana-Ecuador. Email: jcueva@ups.edu.ec, ORCID: https://orcid.org/0000-0002-3055-1060

** Máster en Administración de Empresas. Profesor-Investigador de la carrera administración de empresas de la Universidad Politécnica Salesiana del Ecuador sede Guayaquil. Editor de Revista RETOS y miembro de Grupo de Innovación Educativa Mejorando las Prácticas Áulicas (GIE-MPA), Universidad Politécnica Salesiana-Ecuador. Email: nsumba@ups.edu.ec, ORCID: https://orcid.org/0000-0001-7163-4252

*** Máster en negocios internacionales y gestión de comercio exterior. Profesora de la carrera administración de empresas de la Universidad Politécnica Salesiana del Ecuador sede Guayaquil. Universidad Politécnica Salesiana-Ecuador. Email: sdelgadof@ups.edu.ec, ORCID: https://orcid.org/0000-0002-1841-1938 


\title{
Social marketing and its impact on consumer behavior
}

\begin{abstract}
This article seeks to determine the effects of social marketing on consumer buying behavior. For this, the questionnaire was used as a data collection instrument, this tool was subjected to a reliability test obtaining a Cronbach's alpha of 0.815 , which was applied to 432 Ecuadorians. Next, a correlational study was developed between the variables, the result of the investigation demonstrates the existence of a low intensity correlation (Spearman's rho $=.322$ ), result that corroborates that social marketing does not have the ultimate purpose of motivating or positively influencing the consumer's purchase decision, on products that the company sells, however, if it favors the corporate image
\end{abstract}

Keywords: Buying behavior; consumer; corporate image; social marketing

\section{Introducción}

Las empresas u organizaciones comerciales forman parte e interactúan con los diferentes actores de una sociedad, dicho en otras palabras, toda organización comercial está en la obligación de administrar sus relaciones con los actores del entorno lo cual forma un sistema de interacción entre las partes. En este sentido Pantoja-Aguilar y Garza-Treviño (2019) indican que las organizaciones deben tener una visión sistémica o integradora, para lo cual hay que dar mayor importancia a las interacciones con el entorno, y resaltan que un sistema para este caso empresa depende de su relación con otro sistema mayor.

Lo expuesto anteriormente quedó en evidencia con la presencia de la Covid-19 en el mundo, donde las organizaciones comerciales forman parte e interactúan con un sistema mayor, es decir, los gobiernos de los países. Estos dos engranajes principales del sistema debieron aunar esfuerzos para tratar de conseguir el bienestar y la concientización de las sociedades o comunidades, a través de diferentes acciones de responsabilidad social. En este sentido Martin-Fiorino y Reyes (2020) considerando el contexto generado por la pandemia resaltan que las organizaciones comerciales deben dejar de lado la elección entre el mercado objetivo o la comunidad para tomar un papel activo en lo concerniente a su responsabilidad social.

Relacionado, Crane y Matten (2020) afirman que frente al virus $y$ los problemas que trajo consigo, las organizaciones comerciales debieron reformular y adaptar rápidamente sus acciones de responsabilidad social, en primer lugar, observando su filosofía institucional; y, en segundo lugar, considerando las nuevas necesidades y actores que emergieron dentro del entorno impuesto por la pandemia.

Basados en este planteamiento, las actividades empresariales enfocadas 
en el bienestar social en tiempos de pandemia reciben una valoración muy alta por parte de trabajadores, consumidores y clientes, tanto reales como potenciales, situación que mejora sustancialmente la reputación de la empresa, así como la fidelización de la sociedad hacia la marca, factores de suma importancia en el contexto actual ya que de cierto modo aseguran la sostenibilidad económica de la empresa (Jiang y Wen, 2020; Palma-Ruiz, CastilloApraiz y Gómez-Martínez, 2020).

Para Sánchez (2015) y Delfín y Acosta (2016) toda empresa posee como objetivo fundamental buscar los recursos económicos necesarios para su funcionamiento, en este sentido, en tiempos de pandemia las firmas tuvieron que buscar y adaptar estrategias para asegurar su sostenibilidad económica, conociendo que los efectos producidos por el virus han llegado también a sus grupos objetivos. Lo anterior, resulta validado dentro de las acciones de responsabilidad social de las empresas, ya que lograr los recursos necesarios para garantizar la continuidad de las operaciones aparece como una de las principales responsabilidades.

La actual situación se ha presentado como una oportunidad para la aplicación de acciones de marketing social que permitan, por un lado, que la sociedad adopte comportamientos pertinentes para hacer frente a la pandemia, y por otro, que la empresa se beneficie del mejoramiento o repunte en su imagen corporativa, no solo por sus buenas acciones, sino, por el interés real en los problemas que aquejan al mundo, en general, y a las comunidades, en particular. (He y Harris, 2020; Huang y Liu, 2020). Este fortalecimiento de la imagen corporativa, puede generar situaciones económicamente favorables para la empresa según la investigación realizada por Martínez et al. (2018)

Considerando el contexto empresarial descrito $\mathrm{y}$ teniendo en cuenta que el objetivo del marketing social es el cambio del pensamiento o modo de actuar de las personas por uno que las sociedades consideren adecuados para su bienestar, la presente investigación busca determinar la incidencia de las acciones del marketing social sobre el comportamiento de compra del consumidor frente a los productos o servicios que comercializa la empresa, comprendiéndose esto como un posible efecto colateral de este tipo de marketing.

\section{Marketing social y consumidores: consideraciones conceptuales}

Para Stanton, Etzel y Walker (2007), Forero (2010), Kotler y Keller (2012), Kotler y Armstrong (2012) el marketing social abarca un grupo de actividades estratégicas que tienen como finalidad crear valor para generar bienestar en los consumidores y clientes actuales, además de tratar de cambiar y promover el modo de actuar de las personas avalando el bienestar de la sociedad a corto, mediano y largo plazo, al respecto, Fernández et al. (2017), agregan que el marketing social busca incidir en la conducta y cambiar la forma de actuar de los consumidores, clientes y personas de una sociedad por un comportamiento que la comunidad acepte como adecuado para su bienestar.

En la pandemia ocasionada por la Covid-19, esta vertiente del marketing se vuelve necesaria en la gestión organizacional como respuesta a los 
problemas que las sociedades a nivel global vivían y continúan viviendo. En este sentido, se desarrollaron estrategias, acciones y mensajes de concientización que apuntan a mantener el distanciamiento social, el uso de la mascarilla y la constante desinfección de las manos; estos procederes continúan siendo promovidos por diferentes marcas a nivel global; con la finalidad de instaurar en la sociedad, comportamientos necesarios para convivir en tiempos de pandemia (Alkhatib, 2020; Lee, 2020).

Estas acciones debieron ser adoptadas rápidamente por las organizaciones comerciales quienes estaban obligadas a promover el bienestar de la sociedad donde ellas interactúan, a través de programas de salud y de concientización dirigidos a empleados, consumidores, clientes y a la comunidad en general, en este sentido Grijalva y Fernández (2020) agregan que el cumplimiento de este tipo de acciones de responsabilidad social permitirá a la organización consolidar y sostener una buena imagen en el entorno.

Junto con los problemas de salud que contrajo la presencia de la Covid-19, la Comisión Económica para América Latina y el Caribe [CEPAL] (2020) menciona que surgen problemas económicos a nivel global, situación que impactó a las organizaciones, las cuales debieron diseñar diferentes estrategias. Katz, Jung y Callorda (2020), hacen referencia a la adopción de tecnologías digitales para afrontar la crisis. También las empresas debieron adaptar sus estrategias de marketing y adoptar nuevas tácticas de índole social para hacer frente a esta situación y asegurar su existencia, en este sentido Moreno (2020) agrega que el marketing y todas sus vertientes como el marketing social, deben estar en constante adaptación para que las empresas aprovechen las oportunidades que pudieran presentarse, incluso en momentos de crisis.

Colocando el reflector sobre los consumidores, las prácticas de marketing social bien coordinadas y fundamentadas parecen tener una incidencia positiva sobre el comportamiento y concientización del consumidor en diversas aristas: salud (Bryant et al. 2000; Gordon et al. 2006), desarrollo sostenible y cuidado del medio ambiente (Tabanico y Schultz, 2007; Kennedy, 2010; McKenzie-Mohr, et al. 2012), transporte y comunicaciones (Cooper, 2007; Thogersen, 2014). En este sentido, los consumidores esperan que las empresas presenten una congruencia entre sus propuestas con valores relevantes para la sociedad. De esta forma, la evaluación y selección de alternativas que realiza el consumidor pueden basarse en si las organizaciones actúan de forma consistente en la búsqueda del bienestar de la sociedad (Forte y Lamont, 1998).

Justamente es allí donde el marketing tradicional se distancia del marketing social, dado que este último está direccionado a la sociedad en su conjunto o a una parte de ella, está enfocado en solucionar problemas de tipo colectivo, siempre en busca del bienestar de la comunidad y a favor del grupo humano abarcado (Páramo, 1993). Por ello es que el marketing social en algunas ocasiones, busca persuadir al consumidor, mientras que, en otras, disuadirlo. Se busca persuadir al consumidor de que adopte acciones o comportamientos en pro de su beneficio; por ejemplo: realizar actividades deportivas, consumir alimentos saludables como parte de su dieta alimenticia. Por otra parte, la disuasión, busca que el consumidor abandone o 
disminuya comportamientos negativos, como pueden ser: dejar de fumar cigarrillos, ingerir bebidas alcohólicas en exceso o conducir vehículos si se ha bebido.

Al considerar al marketing social como parte de una estrategia más amplia de cambio social, Lambin (1986) reconoce que este cambio puede ser producido en 4 diferentes niveles, del más sencillo al complejo son: cognoscitivo, acción, comportamiento y valores. El cambio cognoscitivo se presenta en la comprensión y el conocimiento (comprensión de un semáforo nutricional en los alimentos); el cambio en la acción se refleja cuando los individuos realizan una acción específica en un momento de tiempo específico (asistir a votar); el cambio en el comportamiento hace referencia a que los consumidores adopten cierto modelo de conducta (no conducir si se ha bebido o ingerir alimentos saludables); un cambio de valores, claramente lo más complejo, intenta permear en la cultura de una sociedad o comunidad para modificar los valores arraigados y afianzados ante algún concepto o situación.

Por otro lado, los fines empresariales, los medios y recursos para lograrlos impactan negativa o positivamente en el entorno y en la opinión de la sociedad conformada por clientes potenciales y reales quienes pueden responder de forma favorable o no, según recuerden lo que la empresa está realizando. Es, en este punto, donde se unen las acciones del marketing social y la imagen corporativa empresarial. En este sentido, Giuliani et al. (2012); Pinzón (2018) y Justiniano (2020), afirman que las acciones del marketing social contribuyen y tienen un efecto positivo sobre la imagen corporativa de las sociedades comerciales, desde este punto de vista las acciones de marketing social se considerarían una inversión o decisión estratégica para la empresa.

En lo concerniente a la imagen corporativa, Blázquez y Peretti (2012), Pintado y Sánchez (2013), Capriotti (2013) la describen como un mapa mental de la sociedad comercial que forman las personas, clientes 0 consumidores, esto como consecuencia del procesamiento e interpretación de la información referente a la empresa, en este sentido, se puede indicar que la imagen corporativa es la percepción que tienen los clientes y consumidores tanto reales como potenciales frente a las actividades empresariales (Ramos y Valle, 2020), percepción que puede incentivar, promover o condicionar el uso o consumo de productos o servicios.

Con relación a las variables del marketing social, aparentemente la ejecución de estrategias solo involucraría el uso de acciones publicitarias; no obstante, conviene indicar que va más allá, se debe incluir cualquier elemento estratégico que permita alcanzar la adaptación de un pensamiento social adecuado para el bienestar de la población (Kotler y Armstrong, 2012). En relación, se analizan las investigaciones de Pérez (2004); Forero (2010); Santesmases (2012); Ahmadi et al. (2017) aportan a la construcción y descripción de las variables del marketing social las siguientes definiciones:

- Producto Social: Para CárdenasRebelo y Orozco-Toro (2020) el producto se debe considerar como un intangible pues se transforma en creencias, valores o actitudes que debe asumir el grupo objetivo, tiene como finalidad que los integrantes de la sociedad se apropien o se empoderen de la iniciativa social. Por ello, las organizaciones en 
tiempos de pandemia debieron diseñar este tipo de intangibles con el objetivo de generar conciencia y predisposición al cumplimiento de normativas sanitarias por parte de sus grupos objetivos, situación valorada positivamente y de forma general por la sociedad.

- Precio Social: Olivera y Pulido (2018), señalan que en el caso del precio no debe hacerse una analogía con la consecución de valores monetarios., Esta situación se justifica dado que el marketing social no persigue beneficios económicos, sino el cambio y adopción de comportamientos alineados al bienestar de la sociedad, sin embargo indirectamente como ya se ha explicado, un efecto del marketing social es el mejoramiento de la imagen corporativa, lo cual podría considerarse como una retribución hacia la empresa por parte de la sociedad, y que a su vez podría beneficiar los intereses económicos de la empresa.

- Punto de Venta Social: Las personas que accedan al cambio de pensamiento o modo de actuar promovido deben poseer todos los medios necesarios para la continuidad del cambio, para ello se vuelve necesario la participación de los diferentes actores de la sociedad, gobierno, empresas públicas y privadas así también la unión de diferentes grupos influyentes (Olivera y Pulido, 2018).

- Publicidad social: Esta P incluye la correcta transmisión del mensaje por diferentes medios según el grupo objetivo seleccionado, sin olvidar que el mensaje no debe buscar la venta de un producto o servicio específico de la empresa. Cárdenas-
Rebelo y Orozco-Toro (2020) en su investigación mencionan que publicidad social comprende "Un esfuerzo organizado, dirigido por un grupo (el agente de cambio), que intenta persuadir a otros (adoptantes objetivo) de que acepten, modifiquen, o abandonen ciertas ideas, actitudes, prácticas y conductas".

Todo lo expuesto anteriormente deja claro que marketing social no es la comercialización de productos, servicios o el desarrollo de actividades que den paso a la generación directa de utilidades económicas para la empresa; sin embargo, la aplicación de estrategias de marketing social tiene un impacto positivo en la imagen y reputación de la empresa frente a la sociedad, efecto que podría incidir indirecta y positivamente en la conducta de compra de los consumidores o clientes. En este sentido Cueva, Sumba y Villacrés (2020) reflexionan indicando que los líderes empresariales deben estudiar los diferentes factores exógenos $y$ endógenos que pudieran incidir en el modo de actuar del consumidor a favor de las organizaciones.

Por otra parte, es importante enfatizar que el consumidor constantemente cambia su comportamiento y conductas en función de conocimientos adquiridos, experiencias, recomendaciones, así como de las condiciones del entorno en que se desarrolla, es por ello que la pandemia generada por la Covid-19 aparte de los efectos devastadores en salud y economía a nivel mundial, también ha servido como fuente propulsora de cambios en el comportamiento de compra de los consumidores (OrtegaVivanco, 2020; Casco, 2020). 


\section{Consideraciones metodológicas}

El trabajo de investigación realizado es de tipo correlacional, no experimental, transversal y con enfoque cuantitativo. Considerando los resultados de la revisión de literatura realizada en la primera sección, se construyó la operacionalización de las variables (marketing social y comportamiento de compra del consumidor), la cual se muestra en la Tabla 1.

\section{Tabla 1}

\section{Operacionalización de variables}

\begin{tabular}{cc} 
Variable & Dimensiones \\
\cline { 2 - 2 } Marketing Social (independiente) & Producto Social \\
\cline { 2 - 2 } & Precio Social \\
\cline { 2 - 2 } Comportamiento de compra del consumidor & Punto de venta Social \\
\cline { 2 - 2 } (dependiente) & Publicidad Social \\
\cline { 2 - 2 } & Factores Internos \\
\cline { 2 - 2 } & Búsqueda de Información \\
\cline { 2 - 2 } & Decisión de Compra \\
\hline
\end{tabular}

Fuente: Elaboración propia

Se consideró como población a los ecuatorianos económicamente activos, quienes según el Instituto Nacional de Estadística y Censos INEC (2021), hasta febrero de 2021 eran 8.249.661, dado que esta población supera los 100.000 individuos, según Triola (2008) se la puede considerar como población infinita. Como parte del diseño metodológico se consideró un nivel de confianza de $95 \%$ y un error máximo permitido de $5 \%$, obteniendo como resultado: 385 ecuatorianos; sin embargo, se procesaron 432 encuestas, lo cual superó la cantidad de encuestados definida en el diseño metodológico lo que implica un margen de error de $4.71 \%$,

Se utilizó como herramienta de recolección de datos, un cuestionario, el mismo fue diseñado con 16 preguntas: 4 preguntas de tipo sociodemográfico y 12 preguntas para medir la relación entra las dos variables de interés, utilizando como opciones de respuesta la escala de medición de Likert, cabe indicar que el cuestionario fue aplicado durante los meses de febrero y marzo de 2021. Para comprobar la fiabilidad del instrumento se aplicó el test de alfa de Cronbach obteniendo un coeficiente de 0.815 , lo que indica según Hernández, Fernández 
y Baptista (2014), que la confiabilidad del instrumento es elevada.

Para demostrar evidencia estadística de correlación entra las variables de marketing social y comportamiento de compra del consumidor, según la finalidad de la investigación se realizó un análisis bivariante de tipo correlacional, considerando que las dos variables de interés son de escala de medición ordinal se utilizará el coeficiente de correlación de Spearman (rho). Para esto se planteó el siguiente sistema de hipótesis:

Hipótesis nula No existe correlación entre el marketing social y el comportamiento de compra del consumidor.

Hipótesis alternativa $\mathrm{Si}$ existe correlación entre el marketing social y el comportamiento de compra del consumidor.

Se rechazará la hipótesis nula cuando el $p$-valor sea $<0.05$

\section{Marketing social y su incidencia en el comportamiento del consumidor: Resultados}

A continuación, en la tabla 2 se presentan los estadísticos descriptivos más relevantes de la muestra, que permitirán valorar posibles sesgos en la distribución que puedan afectar a la interpretación de los resultados.

Un primer sesgo que se evidencia es la presencia de una mayor cantidad de respuestas por parte de personas que pertenecen a la generación millennial, quienes están con edades comprendidas entre 21 y 44 años, esto se hace relevante ya que según Kotler y Keller (2012), las preferencias de uso y consumo difieren entre las distintas generaciones. También se observa, que la muestra estuvo altamente conformada por personas que poseen estudios universitarios y que pertenecen a un estrato social de clase media.

Tabla 2

Distribución de frecuencias y porcentajes de las variables sociodemográficas del estudio

\begin{tabular}{lcc}
\hline Edad & Frecuencia & Porcentaje \\
\hline Menor a 18 años & 8 & $1.9 \%$ \\
\hline De 18 a 20 años & 56 & $13 \%$ \\
\hline De 21 a 44 años & 292 & $67.6 \%$ \\
\hline Mayor a 44 años & 76 & $17.6 \%$ \\
\hline
\end{tabular}


Cueva Estrada, Jorge Manuel; Sumba Nacipucha, Nicolás Armando; Delgado Figueroa, Stella Paola

Marketing social y su incidencia en el comportamiento del consumidor

Cont... Tabla 2

\begin{tabular}{lcc}
\hline Educación & Frecuencia & Porcentaje \\
\hline Postgrado completo & 136 & $31.5 \%$ \\
\hline Postgrado incompleto & 20 & $4.6 \%$ \\
\hline Secundaria completa & 20 & $4.6 \%$ \\
\hline Sin estudios & 4 & $0.9 \%$ \\
\hline Universidad Completa & 76 & $17.6 \%$ \\
\hline Universidad incompleta & 176 & $40.7 \%$ \\
\hline Clase Social & Frecuencia & Porcentaje \\
\hline Baja & 12 & $2.8 \%$ \\
\hline Media & 300 & $69.4 \%$ \\
\hline Media alta & 44 & $10.2 \%$ \\
\hline Media baja & 76 & $17.6 \%$ \\
\hline Total & 432 & $100 \%$ \\
\hline
\end{tabular}

Fuente: Elaboración propia

La tabla 3 muestra los incidencia al momento de decidir una resultados donde los encuestados compra.

expresaron que factor tiene mayor

\section{Tabla 3}

\section{Factores de incidencia en la decisión de compra del consumidor}

\begin{tabular}{|c|c|c|}
\hline Factor & Frecuencia & Porcentaje \\
\hline Calidad & 152.0 & $35.2 \%$ \\
\hline Cantidad de producto & 16.0 & $3.7 \%$ \\
\hline $\begin{array}{l}\text { Empresas que transmitan men- } \\
\text { sajes de concientización contra la } \\
\text { Covid-19 }\end{array}$ & 8.0 & $1.9 \%$ \\
\hline Marca & 8.0 & $1.9 \%$ \\
\hline Precio & 180.0 & $41.7 \%$ \\
\hline Promociones & 68.0 & $15.7 \%$ \\
\hline Total & 432 & $100 \%$ \\
\hline
\end{tabular}

Fuente: Elaboración propia 
Como se puede observar, el factor que más incide en el comportamiento de compra, según la percepción de los encuestados es el precio, con una representatividad del $41.7 \%$; seguido de la calidad con un $35.2 \%$, factores que los clientes o consumidores asocian según sus pensamientos y percepciones al momento de decidir una compra, estos resultados se ven soportados por diversas investigaciones (Lichtenstein, Ridgway y Netemeyer, 1993; Castro y Rosemberg, 2000; Moreno, 2014). En este punto, se puede agregar lo indicado por Andrés, Gómez y Mondéjar-Jiménez (2015) quienes resaltan la importancia del precio, indicando que la percepción de un precio justo en los consumidores, les permitirá alcanzar mayores niveles de satisfacción, ser consumidores más leales y obtener una mayor confianza en las decisiones tomadas. Finalmente, Matellanes, Villota y Fernández (2018) sostienen que el precio y la calidad son factores que motivan e influyen en la decisión de compra de los consumidores y sostienen que estos factores son primordiales para sostener las ventas de las marcas blancas.

Se totalizaron las respuestas a las 8 preguntas correspondientes a cada una de las 4 dimensiones de la variable marketing social; así como las respuestas a las 4 preguntas ligadas a las 4 dimensiones de la variable comportamiento de compra del consumidor, los totales fueron sometidos al test de normalidad de Kolmogorov Smirnov, el cual mostró como resultado la ausencia de normalidad en todas las dimensiones de las variables. Por lo que, para medir la correlación entre cada una de estas dimensiones y el comportamiento de compra del consumidor, se empleó el test de correlación (rho) de Spearman. El análisis estadístico de las correlaciones se muestra en la tabla 4.

\section{Tabla 4}

\section{Rho de Spearman entre las dimensiones del marketing social y el comportamiento de compra del consumidor.}

\begin{tabular}{lcccc}
\hline Dimensión & Producto & Precio & Punto de venta & Publicidad \\
\hline $\begin{array}{c}\text { Comportamiento de compra del } \\
\text { consumidor }\end{array}$ &, $254^{* *}$ &, $243^{* *}$ &, $306^{* *}$ &, $263^{* *}$ \\
\hline$* *$ La correlación es significativa en el nivel 0,01 (bilateral). \\
$\begin{array}{l}\text { Fuente: Elaboración propia } \\
\text { Fula }\end{array}$
\end{tabular}

Como se puede observar existe la presencia de correlación entre cada una de las dimensiones de marketing social y el comportamiento de compra del consumidor, sin embargo, según Hernández et al. (2003), la intensidad de estas correlaciones es baja.

Para finalizar el estudio correlacional, se sumó el total de las dimensiones: producto, precio, punto de venta y publicidad social, con la finalidad de obtener un resultado integral de la variable marketing social y analizar su posible correlación con la variable comportamiento de compra del consumidor. Para tener una primera apreciación se realizó un gráfico de dispersión entre las variables, como se muestra en el gráfico 1. 


\section{Gráfico 1}

\section{Dispersión entre las variables de marketing social y comportamiento de compra del consumidor.}

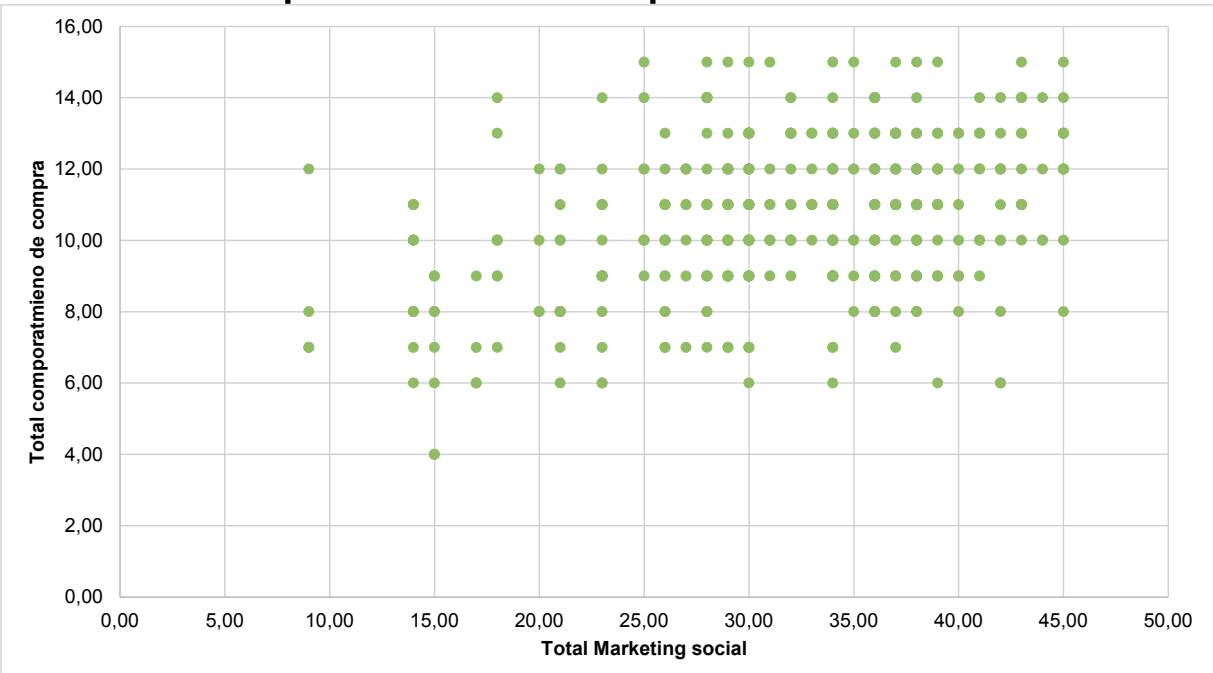

Fuente: Elaboración propia

La nube de puntos que se observa en el gráfico 1 refleja la tendencia positiva entre las variables, esto quiere decir que mientras aumenta el total de la variable marketing social (independiente), la variable comportamiento de compra del consumidor (dependiente) también lo hace. Para conocer la intensidad de la correlación entre las variables se realizó el test de Spearman, el análisis estadístico se presenta en la tabla 5.

\section{Tabla 5}

\section{Rho de Spearman entre los totales de marketing social y comportamiento de compra del consumidor.}

\begin{tabular}{|c|c|c|}
\hline \multicolumn{2}{|c|}{ Rho de Spearman } & Total Marketing social \\
\hline \multirow{3}{*}{$\begin{array}{l}\text { Total comportamiento de compra } \\
\text { del consumidor }\end{array}$} & Coeficiente de correlación &, $322^{* *}$ \\
\hline & Sig. (bilateral) & 0 \\
\hline & $\mathrm{N}$ & 432 \\
\hline
\end{tabular}

**. La correlación es significativa en el nivel 0,01 (bilateral).

Fuente: Elaboración propia 
Al obtener el $p$ valor $<0.01$ se puede rechazar la hipótesis nula $\mathrm{H}_{0}$; por lo que se acepta la hipótesis alternativa $\mathrm{H}_{1}$, la cual señala la existencia de correlación entre las variables. Mientras que el coeficiente de rho de Spearman de .322 muestra la existencia de correlación positiva baja entre las variables.

En la revisión de literatura no se hallaron investigaciones que aborden directamente la incidencia del marketing social en el comportamiento de compra del consumidor, sobre los productos que comercializa la empresa que desarrolla este tipo de acciones de marketing, sin embargo, existen varias investigaciones que señalan que las estrategias de esta vertiente del marketing permiten que las empresas obtengan una mejora en su imagen corporativa (Giuliani et al. 2012; Pinzón, 2018; Justiniano, 2020).

\section{Conclusiones}

La presencia del coronavirus covid-19 ha afectado negativamente diferentes aspectos de la sociedad, frente a esta situación las organizaciones comerciales debieron activar mecanismos de ayuda para el entorno social en donde desarrollan sus actividades, a través de iniciativas enmarcadas en el contexto de la responsabilidad social empresarial, que beneficien directa o indirectamente a sus stakeholders.

En este sentido, las organizaciones también diseñaron estrategias de marketing social con la finalidad de concientizar a la sociedad sobre los riesgos, responsabilidades y buenas prácticas para combatir la presencia del virus, y de esta manera, tratar de mitigar su impacto negativo.

Si bien es cierto, el leitmotiv del marketing social es intentar cambiar el actuar de las personas, por un comportamiento que busque el bienestar de la sociedad; también es cierto que la aplicación de esta vertiente del marketing construye una imagen corporativa sólida y responsable en la sociedad, la cual podría ser aprovechada por las organizaciones para asegurar su sostenibilidad económica, como un efecto colateral positivo que pudiera incidir en el comportamiento de compra del consumidor.

El análisis estadístico demostró la existencia de correlación con intensidad baja entre las variables: marketing social y comportamiento de compra del consumidor, resultado que es relevante para las empresas si se considera que el marketing social no tiene como fin último motivar o incidir positivamente en la decisión de compra del consumidor, sobre productos que comercializa la empresa; sin embargo, este resultado puede ser analizado y aprovechado por las organizaciones comerciales para diseñar estrategias de marketing social e incluirlas en sus planificaciones anuales. Esta correlación se puede atribuir a que el consumidor tiene la percepción de que las empresas que desarrollan este tipo de estrategias, están interesadas en el bienestar de la sociedad en la que desarrollan sus actividades productivas, y no solo persiguen fines lucrativos, lo cual mejora su imagen corporativa, pudiendo constituirse en un escenario positivo para los intereses económicos de las organizaciones comerciales.

En las preguntas referentes a la variable de marketing social, más del $55 \%$ de los encuestados inclinaron sus opciones de respuesta a los valores más altos, lo cual demuestra una mejor percepción de la imagen corporativa por parte de los consumidores hacia las marcas que muestren más interés 
Marketing social y su incidencia en el comportamiento del consumidor

en mitigar los problemas que pueden estar presentes en la sociedad, sin embargo estos mismos consumidores perciben las estrategias de marketing social como tácticas provenientes de la empresa con la finalidad de incrementar sus ventas, escenario que permite comprender el bajo nivel de correlación entre las variables. A esto se puede añadir que la situación económica generada por la pandemia obliga a los consumidores a restringir aún más el uso de sus recursos económicos, buscando productos y servicios que satisfagan sus necesidades a un precio mínimo con una calidad óptima.

Debido a que el presente estudio se realizó con la presencia del coronavirus en el contexto mundial durante los años 2020 y 2021 y, considerando que el análisis estadístico demostró la existencia de correlación con intensidad baja entre las variables de estudio; sería interesante desarrollar una nueva investigación correlacional una vez que se haya dejado atrás la pandemia y sus efectos devastadores en la sociedad, para de esta forma poder determinar si existe una variación en la intensidad de la correlación.

\section{Referencias Bibliográficas}

Ahmadi, S., Ardabili, H., Haghdoost, A., Nakhaee, N., \&Shams, M. (2017). Promoting physical activity in Iranian women: A qualitative study using social marketing. Electronic Physician, 9(9), 5279-5286. https:// dx.doi.org/10.19082/5279

Alkhatib , A. (2020) Social marketing and social distancing: Jordan experience in combating COVID-19. Dialogoconferences\&journal, 6(2), 33-43. https://repository.globethics.net/ handle/20.500.12424/4022975
Andrés, M., Gómez, M., \& MondéjarJiménez, J. (2015). Comportamiento del consumidor español ante la percepción de precios en hoteles online. Revista de Ciencias Sociales (Ve), XXI(3),311-320. https://doi. org/10.31876/rcs.v21i3.25733

Blázquez, M., y Peretti, M., (2012). Modelo para gestionar la sustentabilidad de las organizaciones a través de la rentabilidad, adaptabilidad e imagen. Estudios Gerenciales, 28(125), 4050. https://www.redalyc.org/articulo. oa?id=21226279005

Bryant, C. A., Forthofer, M. S., Brown, K. R. M., Landis, D. C., y McDermott, R. J. (2000). Community-based prevention marketing: The next step in disseminating behavior change. American Journal of Health Behavior, 24, 61-68. https://doi.org/10.5993/ AJHB.24.1.9

Capriotti, P. (2013). Planificación Estratégica de la Imagen Corporativa. Edita: IIRPInstituto de Investigación en Relaciones Públicas. Málaga, España.

Cárdenas-Rebelo, A., y Orozco-Toro J.A (2020). Percepción de las campañas sociales de prevención de accidentes de tránsito en Ecuador. Retos Revista de Ciencias de la Administración y Economía, 10(20), 219-231. https:// doi.org/10.17163/ret.n20.2020.02

Casco, A. (2020). Efectos de la pandemia de COVID-19 en el comportamiento del consumidor. Innovare: Revista De Ciencia Y Tecnología, 9(2), 98-105. https://doi.org/10.5377/innovare. v9i2.10208

Castro Herrera, S., \& Rosenberg, A. (2000). Precios psicológicos. Análisis de la Percepción del Consumidor. Quipukamayoc, 7(13), 83-92. https:// doi.org/10.15381/quipu.v7i13.5774

Comisión Económica para América 
Latina y el Caribe CEPAL (2020). Contracción de la actividad económica de la región se profundiza a causa de la pandemia: Caerá -9,1\% en 2020. https://bit.ly/2XmFYo2

Cooper, C. (2007). Successfully changing individual travel behavior: Applying community-based social marketing to travel choice. Transportation Research Record. Journal of the Transportation Research Board - The National Academies, 89-99. https:// doi.org/10.3141/2021-11

Crane, A., y Matten, D. (2020). COVID-19 and the future of CSR research. Journal of Management Studies, 58(1). $\quad$ https://doi.org/10.1111/ joms. 12642

Cueva-Estrada, J., Sumba-Nacipucha, N., \& Villacrés-Beltrán, F. (2020). El marketing de influencias y su efecto en la conducta de compra del consumidor millennial. Suma de negocios, 11(25), 99-107. http://doi. org/10.14349/sumneg/2020.V11. N25.A1

Delfín, J., y Acosta, M., (2016). Importancia y análisis del desarrollo empresarial. Pensamiento $y$ gestión, 40, 184-202. http://dx.doi. org/10.14482/pege.40.8810

Fernández, A., Pérez, C., Méndez, V., Fernández, C., Méndez, A., y Calero, S. (2017). Marketing social y su influencia en la solución de problemas de salud. Revista Cubana de Investigaciones Biomédicas, 36(3), 1-11. https://bit.ly/2OtYnOB

Forero, J. A. (2010). El marketing social como estrategia para la promoción de la salud. La sociología en sus escenarios, (20). https://revistas. udea.edu.co/index.php/ceo/article/ view/7021

Forte, M., y Lamont, B. T. (1998). The Bottom Line Effects of Greening:
Implications of Environmental Awareness. Academy of Management, 12, 89-90. https://doi. org/10.5465/ame.1998.254983

Giuliani A., Monteiro T., Zambon M., Betanho C., y Lima L (2012). El marketing social, el marketing relacionado con causas sociales y la responsabilidad social empresarial. Invenio, 15(29), 1127. https://www.redalyc.org/articulo. oa? id =87724146003

Grijalva Morejón, B.R., y Fernández Lorenzo, A. (2020). Responsabilidad social empresarial y competitividad en las clínicas de salud privadas de Quito, Ecuador. Cooperativismo y desarrollo, 8(2), 315-328. http:// scielo.sld.cu/pdf/cod/v8n2/2310340X-cod-8-02-315.pdf

Gordon, R., McDermott, L., Stead, M., y Angus, K. (2006). The effectiveness of social marketing interventions for health improvement: What's the evidence? Public Health, 120, 11331139. https://pubmed.ncbi.nlm.nih. gov/17095026/

He, H., y Harris, L. C. (2020). The Impact of Covid-19 Pandemic on Corporate Social Responsibility and Marketing Philosophy. Journal of Business Research, 116, 176-182. https://doi. org/10.1016/j.jbusres.2020.05.030

Hernández, R., Fernández, C. y Baptista, M. (2003). Metodología de la investigación. Editorial McGrawHill.

Huang, H., y Liu, S. (2020). "iDone para ayudar a combatir el COVID-19!" ¿Cómo afecta la tipografía a la eficacia del marketing de RSE? International Journal of Contemporary Hospitality Management, 32(10), 3315-3333. https://doi.org/10.1108/ IJCHM-05-2020-0462

Instituto Nacional de Estadística y Censos 
Cueva Estrada, Jorge Manuel; Sumba Nacipucha, Nicolás Armando; Delgado

Figueroa, Stella Paola

Marketing social y su incidencia en el comportamiento del consumidor

INEC (2021). Encuesta Nacional de Empleo, Desempleo y Subempleo 2021. https://www.ecuadorencifras. gob.ec/documentos/ web-inec/EMPLEO/2021/ Febrero-2021/202102 Mercado Laboral.pdf

Jiang, Y., y Wen, J. (2020). Effects of COVID-19 on hotel marketing and management: A perspective article. International Journal of Contemporary Hospitality Management, 32(8), 2563-2573. https://doi.org/10.1108/ IJCHM-03-2020-0237

Justiniano, D. (2020). Marketing social y responsabilidad social corporativa. Oikos Polis, 5(1), 5183. https://www.scielo.org.bo/scielo. php? script=sci arttext\&pid=S241522502020000100004\&lng=es\&tlng $=$ es

Katz, R., Jung, J., \&Callorda, F. (2020). El estado de la digitalización de América Latina frente a la pandemia del COVID-19. CAF. https://bit. ly/3k3109F

Kennedy, A. L. (2010). Using communitybased social marketing techniques to enhance environmental regulation. Sustainability, 2, 1138-1160.

Kotler, P., y Armstrong, G. (2012). Fundamentos de Marketing. 11ma. ed. Naucalpan de Juárez. Pearson Educación de México.

Kotler, P., y Keller, K. (2012). Dirección de Marketing. 14 ed. Pearson Educación de México.

Lambin, J. J. (1986). Le marketing stratégique: fondements, méthodes et applications. McGraw-Hill

Lee, N. (2020). Reducing the Spread of COVID-19: A Social Marketing Perspective. Social Marketing Quarterly, 26(3), 259-265. https://doi. org/10.1177/1524500420933789
Lichtenstein, D. Ridgway, N y Netemeyer, R. (1993). Price Perceptions and Consumer Shopping Behavior: A Field Study. https://bit.ly/3AbB13b

Martin-Fiorino, V., \& Reyes, G. E. (2020). Desafíos y nuevos escenarios gerenciales como parte de la herencia del Covid-19. Revista Venezolana De Gerencia, 25(90), 722-734. https:// doi.org/10.37960/rvg.v25i90.32413

Martínez Escareño, I. M., Casillas Rancurello, M. F., Núñez Alfaro, C. M., González Galindo, A. D., Aguilera Valdez, A. E., \& Portales, L. (2018). Influencia del marketing social y prácticas de RSE en la intención de compra de los millennials. Revista Universidad y Empresa, 20(35), 251-280. https:// doi.org/10.12804/revistas.urosario. edu.co/empresa/a.6034

Matellanes Lazo, M., Villota Mancebo, A., \& Fernández Merino, R. (2018). Imagen de las marcas blancas en el sector alimentario según la perspectiva del consumidor español. Revista De Comunicación De La SEECI, (45), 103$120 . \quad$ https://doi.org/10.15198/ seeci.2018.45.103-120

McKenzie-Mohr, D., Schultz, W. P., Lee, N. R., \& Kotler, P. (2012). Social marketing to protect the environment: What works. Thousand Oaks, CA: Sage.

Moreno, D. (2020) Marketing social y responsabilidad social corporativa. Oikos Polis, Revista latinoamericana de Ciencias Económicas y Sociales, 5(1), 39-71. http://www.scielo.org.bo/ pdf/rlces/v5n1/v5n1_a04.pdf

Moreno, H. (2014). La influencia del precio y las estrategias de comunicación visual basadas en simbología cultural sobre la preferencia de marcas ecológicas y consumo sostenible. Revista Escuela de Administración 
de Negocios, 77, 168-183. https:// doi.org/10.21158/01208160. $\underline{\mathrm{n} 77.2014 .822}$

Olivera, E.S., \& Pulido, V.M.. (2018). Marketing social: Su importancia en la resolución de problemas sociales. Revista Científica de la UCSA, 5(2), 26-35. https://dx.doi.org/10.18004/ ucsa/2409-8752/2018.005(02)026$\underline{035}$

Ortega-Vivanco, M. (2020). Efectos del Covid-19 en el comportamiento del consumidor: Caso Ecuador. Retos Revista de Ciencias de la Administración y Economía, 10(20), 233-247. https://doi.org/10.17163/ ret.n20.2020.03

Palma-Ruiz, J. M., Castillo-Apraiz, J., y Gómez-Martínez, R. (2020). Socially responsible investing as a competitive strategy for trading companies in times of upheaval amid COVID-19: Evidence from Spain. International Journal of Financial Studies, 8, 41, 1-13. https://doi. org/10.3390/ijfs8030041

Pantoja-Aguilar, M. P., y GarzaTreviño, J. R. (2019). Etapas de la administración: hacia un enfoque sistémico. Revista Escuela de Administración de Negocios, (87), 139-154. DOI: https:// doi.org/10.21158/01208160. $\underline{\mathrm{n} 87.2019 .2412}$

Páramo, D. (1993). International strategic marketing for a regio. A Colombiancas. Tesis de doctorado, Universithé Catolic de Louvain. Louvain-LaNeuve, e Bélgica).

Pérez, L. (2004). Marketing social: Teoría y práctica. Pearson Educación de México.

Pintado, T. y Sánchez, J. (2013). Imagen Corporativa, Influencia en la gestión empresarial. 2da. ed. ESIC Editorial.
Pinzón, G. (2018), Marketing social para el fortalecimiento de la imagen corporativa de los organismos públicos del municipio Maracaibo. Marketing visionario, 6 (2), 2240. https://ojs.urbe.edu/index.php/ market/article/view/1644

Ramos, E., y Valle, N. (2020). Gestión de imagen corporativa como estrategia de sostenibilidad: camino al cambio empresarial. Revista Universidad y Sociedad, 12(1), 292-298. https://scielo.sld.cu/scielo. php? script=sci arttext\&pid=S2218$\underline{36202020000100292 \& \operatorname{lng}=\text { es\&tlng= }}$ es

Sánchez, V. (2015). La redefinición del papel de la empresa en la sociedad. Revista Castellano-Manchega de Ciencias Sociales, 20, 129-145. Recuperado de https://www.redalyc. org/pdf/3221/322142550008.pdf

Santesmases, M. (2012). Marketing: Conceptos y estrategias. 6ta. ed. Ediciones Pirámide.

Stanton, W., Etzel, M., Walker, B. (2007). Fundamentos de Marketing 14 ed. McGraw-Hill.

Tabanico, J. J., y Schultz, W. P. (2007). Community-Based Social Marketing. BioCycle, 8, 41-44. https://www. biocycle.net/community-basedsocial-marketing/

Thogersen, J. (2014) Social Marketing in Travel Demand Management. In: Gärling T., Ettema D., Friman M. (eds) Handbook of Sustainable Travel. Springer, Dordrecht. https:// doi.org/10.1007/978-94-007-703488

Triola, M. (2008). Estadística. 10ma. Ed. Pearson Educación. 